\title{
Large and very large scale motions in rough- bed open-channel flow
}

\author{
Stuart Cameron ${ }^{1,}{ }^{*}$, Vladimir Nikora ${ }^{1}$, Mark Stewart ${ }^{1}$, and Andrea Zampiron ${ }^{1}$ \\ ${ }^{1}$ School of Engineering, University of Aberdeen, Kings College, Aberdeen, United Kingdom
}

\begin{abstract}
Long duration PIV measurements in rough-bed (glass beads) open-channel flow (OCF) reveal that the pre-multiplied spectra of the streamwise velocity has a bimodal distribution due to the presence of large and very large scale motions (LSMs and VLSMs, respectively). The existence of VLSMs in boundary layers, pipes and closed channels has been acknowledged for some time, but strong supporting evidence for their presence in OCF has been lacking. Length scales of the large and very large scale motions in OCF exhibit different scaling properties; whereas the streamwise length of the LSM scales with the flow depth, the VLSM streamwise length does not scale purely with flow depth and may additionally depend on other scales such as the channel width, roughness height, or viscous length. Supplementary data for flows over self-affine fractal rough beds support these findings and additionally indicate that the length of VLSMs may grow along the extensive distance from the channel entrance. The origin and nature of LSMs and VLSMs are still to be resolved, but differences in their scaling suggest that VLSMs in rough-bed open-channel flows form independently rather than as a spatial alignment of LSMs.
\end{abstract}

\section{Introduciton}

Kim \& Adrian [1] discovered a bimodal distribution in the streamwise velocity premultiplied spectrum of pipe flow and described the two apparent scales as large and very large scale motions. They proposed that very large scale motions (VLSMs) may result from a streamwise alignment of large scale motions (LSMs) which themselves might represent a collection of smaller hairpin shaped vortices. Subsequently, VLSMs have been discovered in other flow types although their origin remains unclear, with other authors proposing that VLSMs could form independently due to large-scale instability (e.g. [2]). In the case of boundary layers, Hutchins \& Marusic [3] introduced the term 'superstructures' although it is possible that these have the same origin as VLSMs albeit different characteristics due to fundamental differences in the boundary conditions of pipe, closed channel, and boundary layer flows. Comparing pre-multiplied spectra across these three flow types, Monty et al. [4] found a remarkable resemblance between pipe and closed-channel flows; boundary layers in comparison exhibit superstructures in the logarithmic layer, being significantly suppressed in the outer layer.

\footnotetext{
* Corresponding author: s.cameron@abdn.ac.uk
} 
Most studies of VLSMs and superstructures in pipes, closed channels, and boundary layers have been undertaken with smooth-wall conditions. Although smooth wall conditions are also relevant to open-channel flows, most practical situations involve roughbed OCFs and thus their study is of the utmost importance. There are many engineering challenges such as the assessment of bed stability, sediment transport, mixing, and hydraulic resistance in rivers and canals that require the advanced understanding of roughbed OCF turbulence. Therefore, the focus of this study is on rough-bed OCF.

The main objective is to identify and quantify VLSMs in open-channel flows over hydraulically fully rough beds represented by glass beads and by self-affine fractal surfaces. In the case of glass beads, the long duration ( 2 hour) particle image velocimetry (PIV) measurements have been used and the results have initially been reported in [5]. In the present paper, these measurements are supplemented with complimentary data from a series of 4-hour duration ADV records obtained in flows over self-affine rough beds. The focus is on OCFs at low and intermediate submergences, which remain the least studied flow configurations in spite of their ubiquitous occurrence in nature and engineering. Section 2 outlines the experimental facility, instrumentation, and procedures, particularly focusing on PIV measurements in flows over glass beads. Section 3 summarises the background hydraulic conditions, bulk statistics, velocity correlations, spectra, and turbulence scales. Finally, the main findings are briefly summarised in the Conclusions (Section 4).

\section{Experimental setup}

\subsection{Open-channel and flow configurations}

Experiments with OCFs over glass beads were conducted in the Aberdeen Open-Channel Facility (AOCF). The facility consists of a $1.18 \mathrm{~m}$ wide and $18 \mathrm{~m}$ long re-circulating openchannel flume and a motorised instrumental carriage which houses a PIV system and supporting instrumentation. The entire bed of the flume and part of the entrance tank (19.2m total length) was covered for these experiments with a single layer of $16 \mathrm{~mm}$ diameter $(D)$ glass spheres. The spheres were precisely aligned in a hexagonally close packed arrangement with the help of perforated stainless steel plates. The installed plates were levelled using shims to within $+/-0.25 \mathrm{~mm}$. Experiments were conducted with relative submergences $(H / D)$ between 1.9 and 7.5 (Table 1) and constant shear velocity $(u *=0.042 \mathrm{~m} / \mathrm{s})$. The roughness Reynolds number $D^{+}=D u * v$ was 605 for all experiments indicating fully rough flow conditions. The friction Reynolds number $H^{+}=H u * / v$ varied between 1140 and 4540. The Froude number was well below 1.0 securing a flat water surface without visible surface waves; measured standard deviations of water surface level varied within $0.28-0.45 \mathrm{~mm}$. The flows were uniform, with water surface slope deviating from the bed surface slope by a maximum of $2.5 \times 10^{-5}$, and steady to within a flowrate standard deviation of $0.0002 \mathrm{~m}^{3} / \mathrm{s}$.

Table 1. Flow conditions used for the experiments. $H$ is flow depth above the roughness tops, $D$ is sphere diameter, $Q$ is flowrate, $S_{0}$ is channel slope, $U=Q / B H$ is the bulk velocity, $B$ is the channel width, $u^{*}=\left(g H S_{0}\right)^{0.5}$ is shear velocity, $R=U H / v$ is the bulk Reynolds number.

\begin{tabular}{|l|l|l|l|l|l|l|l|}
\hline RUN & $H(\mathrm{~mm})$ & $Q\left(\mathrm{~m}^{3} / \mathrm{s}\right)$ & $U(\mathrm{~m} / \mathrm{s})$ & $S_{0}$ & $u^{*}(\mathrm{~m} / \mathrm{s})$ & $R$ & $H / D$ \\
\hline H030 & 30.1 & 0.0153 & 0.431 & 0.00600 & 0.042 & 11700 & 1.9 \\
\hline H050 & 50.3 & 0.0275 & 0.463 & 0.00360 & 0.042 & 21000 & 3.1 \\
\hline H070 & 70.5 & 0.0404 & 0.486 & 0.00257 & 0.042 & 30800 & 4.4 \\
\hline H095 & 94.9 & 0.0569 & 0.508 & 0.00189 & 0.042 & 43400 & 5.9 \\
\hline H120 & 120.1 & 0.0745 & 0.526 & 0.00150 & 0.042 & 56900 & 7.5 \\
\hline
\end{tabular}


To supplement the PIV data over the glass bead roughness type, we have performed additional experiments using a Vectrino ADV in flows over self-affine fractal roughness to check the development of VLSMs along the channel. The self-affine roughness has a random surface with power law spectral slope $\left(\propto k^{-\beta}\right)$ and a Gaussian probability distribution of bed elevations with standard deviation equal to $1.5 \mathrm{~mm}$. The roughness surface was computer-generated over a periodic domain of $392 \times 392 \mathrm{~mm}$, machined on a computer controlled milling device, and replicated using epoxy resin. Three sets of rough surfaces were produced with spectral slopes of $\beta=1,5 / 3$, and 3 . Manufacture and installation of the roughness is further described in [6]. We measured velocity time series for a 4-hour duration every $1.0 \mathrm{~m}$ along the flume at an elevation of $z / H=0.3$. Flow conditions for the experiments are described in Table 2.

Table 2. Flow conditions for the experiments with self-affine roughness (symbols are as in Table 1). The characteristic roughness height $\Delta=6 \mathrm{~mm}$ was selected as four times the standard deviation of the bed surface elevation.

\begin{tabular}{|l|l|l|l|l|l|l|l|}
\hline RUN & $H(\mathrm{~mm})$ & $Q\left(\mathrm{~m}^{3} / \mathrm{s}\right)$ & $U(\mathrm{~m} / \mathrm{s})$ & $S_{0}$ & $u *(\mathrm{~m} / \mathrm{s})$ & $R$ & $H / \Delta$ \\
\hline H120 $\beta=1$ & 119.9 & 0.0412 & 0.291 & 0.00050 & 0.024 & 34903 & 20.0 \\
\hline $\mathrm{H} 080 \beta=1$ & 80.0 & 0.0249 & 0.263 & 0.00076 & 0.024 & 21078 & 13.3 \\
\hline $\mathrm{H} 120 \beta=5 / 3$ & 120.3 & 0.0414 & 0.292 & 0.00050 & 0.024 & 35094 & 20.1 \\
\hline $\mathrm{H} 080 \beta=5 / 3$ & 80.2 & 0.0253 & 0.267 & 0.00076 & 0.024 & 21424 & 13.4 \\
\hline $\mathrm{H} 120 \beta=3$ & 120.2 & 0.0456 & 0.322 & 0.00050 & 0.024 & 38674 & 20.0 \\
\hline $\mathrm{H} 080 \beta=3$ & 80.4 & 0.0289 & 0.304 & 0.00076 & 0.024 & 24449 & 13.4 \\
\hline
\end{tabular}

\subsection{Stereoscopic particle image velocimetry}

A four-camera stereoscopic PIV system was used to measure all three velocity components in a wall-normal transverse plane (Figure 1). The measurement plane covered the full flow depth, extended $330 \mathrm{~mm}$ across the flow and was positioned $12.7 \mathrm{~m}(>100 H)$ downstream from the flume entrance. Images were recorded directly to a fast disk array at a rate of 32 image pairs per second per camera for a continuous duration of 120 minutes at each of the 5 flow configurations identified in Table 1. We use an iterative deformation method (IDM, [7]) to extract velocity fields from the PIV images. Further detail of the system including seeding, calibration and measurement resolution is available in [5].

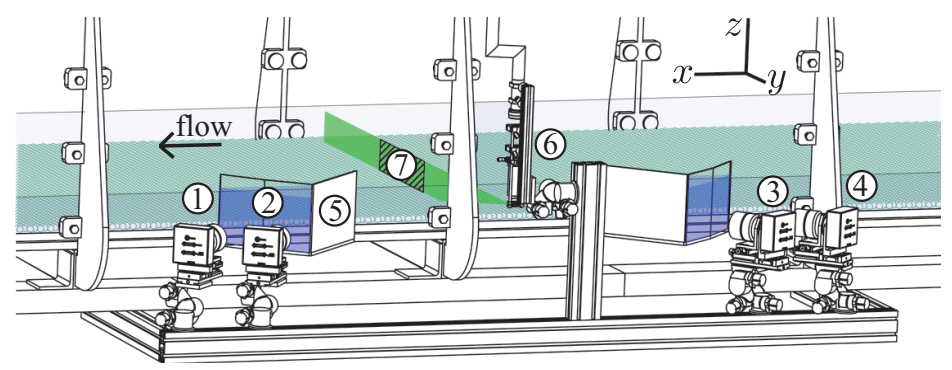

(1) camera 1

(2) camera 2

(3) camera 3

(4) camera 4

(5) water prism

(6) laser optics

(7) measurement window

Fig. 1. Measurement section of the Aberdeen Open-Channel Facility and stereoscopic particle image velocimetry setup. 


\section{Results}

\subsection{Bulk statistics}

Bulk statistics for the studied flow conditions with glass beads on the bed are summarised in Figure 2 and reported with further detail in [5]. The data indicate a logarithmic scaling range for the streamwise velocity near the bed with Kármán coefficient $\kappa=0.38$. The velocity variances show a near-bed region where submergence affects $(H / D)$ are important, particularly for the streamwise velocity component, and the linear distribution of the Reynolds stress confirms near two-dimensionality of the studied flow field.
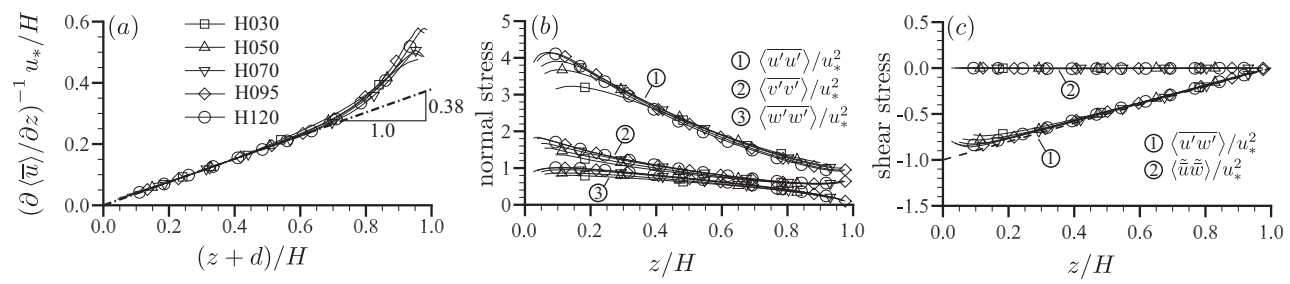

Fig. 2. Mean velocity distribution (a), Reynolds normal stresses (b), and Reynolds shear stress and form-induced stress (c). Symbols are shown every tenth measurement point for clarity.

\subsection{Large scale streaks}

The pseudo-instantaneous velocity field in OCFs over glass beads (Figure 3), reconstructed from time series records using Taylor's frozen turbulence hypothesis, is composed of alternating in the transverse direction and elongated in the streamwise direction regions of high and low momentum fluid visually very similar to the superstructures identified by Hutchins \& Marusic [3] in their boundary layer studies. It is not easy to directly identify length scales of the large streaks from the 'instantaneous' flow fields as they tend to meander, fade, and merge with other features without providing discernible start and end points. We will in the next section use correlation functions and spectra to extract characteristic scales and examine the potential influence of relative submergence.

$$
u^{\prime}(\mathrm{m} / \mathrm{s}) \quad \begin{array}{llllll}
-0.1 & -0.06 & -0.02 & 0.02 & 0.06 & 0.1 \\
& & & & &
\end{array}
$$

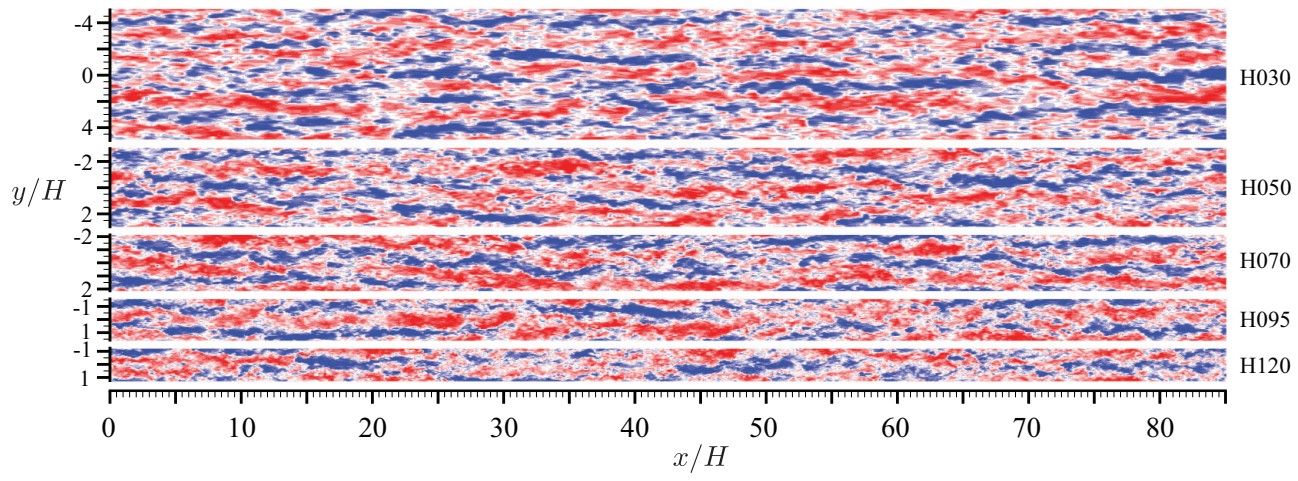

Fig. 3. 'Instantaneous' velocity fluctuations at $z / H=0.5$. 


\subsection{Correlation functions and spectra}

Selected components of the two-point correlation tensor:

$$
C u_{i} u_{j}\left(\Delta y, \Delta z, z_{0}\right)=\frac{\sum^{y} \sum^{t} u_{i}^{\prime}\left(y, z_{0}, t\right) u_{j}^{\prime}\left(y+\Delta y, z_{0}+\Delta z, t\right)}{\left(\sum^{y} \sum^{t}\left[u_{i}^{\prime}\left(y, z_{0}, t\right)\right]^{2}\right)^{0.5}\left(\sum^{y} \sum^{t}\left[u_{j}^{\prime}\left(y+\Delta y, z_{0}+\Delta z, t\right)\right]^{2}\right)^{0.5}}
$$

calculated assuming homogeneity in the transverse direction are plotted in Figure 4 for $z_{0}=0.5 H$; where $t$ is a time index, $z_{0}$ is a reference elevation, and $\Delta y$ and $\Delta z$ are displacement increments. The $-C_{u w}$ and $-C_{u v}$ components when plotted as vectors indicate that fluctuations in the streamwise velocity component are correlated with a pattern of repeating counter-rotating depth-scale cells which may be associated with the large scale streaks identified in Figure 3. Figure 4 shows that with increasing submergence of the glass beads the transverse separation of the cells becomes progressively smaller and the central lobe of the $C_{u u}$ correlation shrinks. The counter rotating depth-scale cells are reminiscent of secondary current flow patterns which appear near side walls in open channels (e.g. [8]). The origin, dynamics, and energetics of secondary currents in OCF are still the subject of ongoing research and it is possible that they form due to a differential alignment of meandering VLSMs caused by the channel sidewalls (see also [9] for discussion of potential links between VLSMs and secondary currents).

To identify the prevailing scales in the flow field over glass beads we used premultiplied wave-number spectra as in other LSM and VLSM studies (e.g. $[1,3,4])$. The pre-multiplied spectra for the streamwise velocity component $\left(k_{x} F_{u u}\left(k_{x}\right) / u *^{2}\right.$, where $k_{x}=2 \pi / \lambda_{x}$ and $\lambda_{x}$ is streamwise wavelength) were calculated in the frequency domain and averaged over $225 \times 32$ second windows with, assuming homogeneity, additional averaging over the transverse extent of the measurement domain. Taylor's frozen turbulence hypothesis was used to transform the frequency spectra to the wavenumber domain using a convection velocity equal to the local mean velocity $\langle\bar{u}\rangle$. The spectra (Figure 5) reveal two characteristic length scales in the outer flow and resemble spectra measured in pipe and closed-channels with smooth wall conditions (e.g. [4]). Following the terminology of Kim \& Adrian [1] we will refer to flow structures associated with the shorter of these length scales as large scale motions (LSMs) and structures associated with the larger length scale as very large scale motions (VLSMs). The length scales determined from spectral peaks may not directly relate to the physical length of turbulent structures due to their transverse meandering [3] but, nevertheless, the spectral length scale provides a convenient and consistent reference to compare with other studies. Near the bed $(z / H<0.1)$ the spectral maps are dominated by a single length scale and experiments with larger $H$ suggest a bifurcation point where the distinct LSM and VLSM scales emerge. Peak amplitudes of the pre-multiplied spectra at the LSM and VLSM wavelengths ( $\lambda_{x_{-} L S M}$ and $\lambda_{x_{-} \text {VLSM }}$ respectively) are approximately the same (Figure $5 \mathrm{f}$ and $5 \mathrm{~g}$ ). Amplitude dependence on flow submergence is observed near the bed consistent with the trend noted earlier for the bulk variance. Combining the normalised LSM and VLSM length scales for all experiments (Figure 5h) it is surprising to find that although estimates of the LSM length scale collapse onto a single curve, the estimates of VLSM length scale are stratified according to flow submergence (or equivalently to flow aspect ratio). 


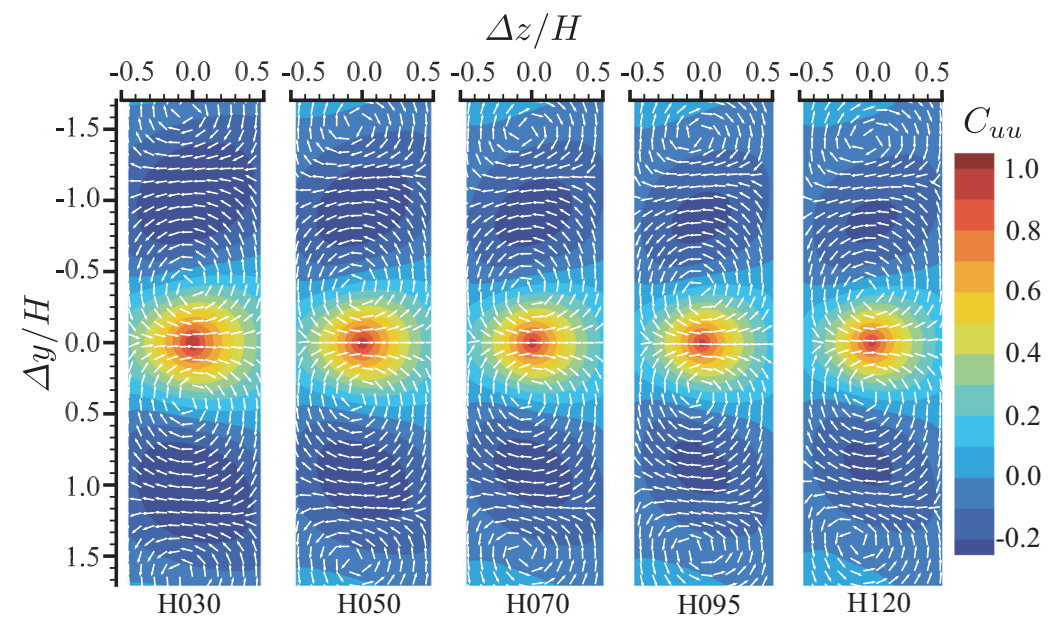

Fig. 4. Two-point correlation functions $C_{u u}, C_{u w}$, and $C_{u v}$ for $z_{0} / H=0.5$. Note that $C_{u w}$ and $C_{u v}$ are shown as vectors $\left(-C_{u w},-C_{u v}\right)$ with unit magnitude.

Plotting the maximum of each $\lambda_{x_{-} V L S M} / H$ distribution (Figure 5i), the VLSMs appear to scale according to MAX $\left[\lambda_{x_{-} V L S M} / H\right]=5.7[B / H]^{0.60}=75[H / D]^{-0.60}=3500\left[H^{+}\right]^{-0.60}$ where given that the ratios $B / D$ and $D u * / v$ are constant for all experiments, all three depth normalisations $\left(B / H, H / D, H^{+}\right)$have equivalent empirical fit. Although further data are required to identify the most appropriate scaling for the VLSMs, we can speculate that $H / D$ scaling does not seem likely given that the smaller LSMs scale independently of the relative submergence (Figure 5h). Reynolds number $\left(\mathrm{H}^{+}\right)$effects have been assessed in other flow types and found to have minimal influence on VLSM length scale (e.g. [10]) though the amplitudes of the pre-multiplied spectra have been found to exhibit Reynolds number dependence [3]. Flow aspect ratio $(B / H)$ is perhaps the most plausible scaling given that $B$ and $\lambda_{x_{-} V L S M}$ are of the same order of magnitude and keeping in mind the potential constraining effect of the flow width. As an alternative to potential $B / H, H / D$, or $H^{+}$mechanisms we consider the role of the development length $x / H$ on the scaling of VLSMs in the following section. Qualitatively similar results are obtained for self-affine roughness some of which are outlined below.

\subsection{Evolution of VLSMs along the channel}

The VLSM length scale extracted from velocity spectra measured over glass beads and selfaffine rough beds is presented as a function of the normalised distance along the channel $(x / H)$ in Figure 6. We observe that the VLSM length scale consistently increases along the channel and for some flow depths may not become fully developed before the end of the channel. This is a surprising result since previous measurements in the same channel have established that bulk statistics of the velocity field typically reach a fully developed condition in approximately 50-100 flow depths. The VLSM length scale for the self-affine roughness appears to be quite similar to that obtained for the glass beads roughness, although the latter data was obtained at fixed $x=12.7 \mathrm{~m}$ but with $x / H$ varying for the different flow depths. We plan to obtain further data at large $x / H$ to establish if the development length can fully explain VLSM length scales or if $B / H, H / D$, or $H^{+}$also play a role. 

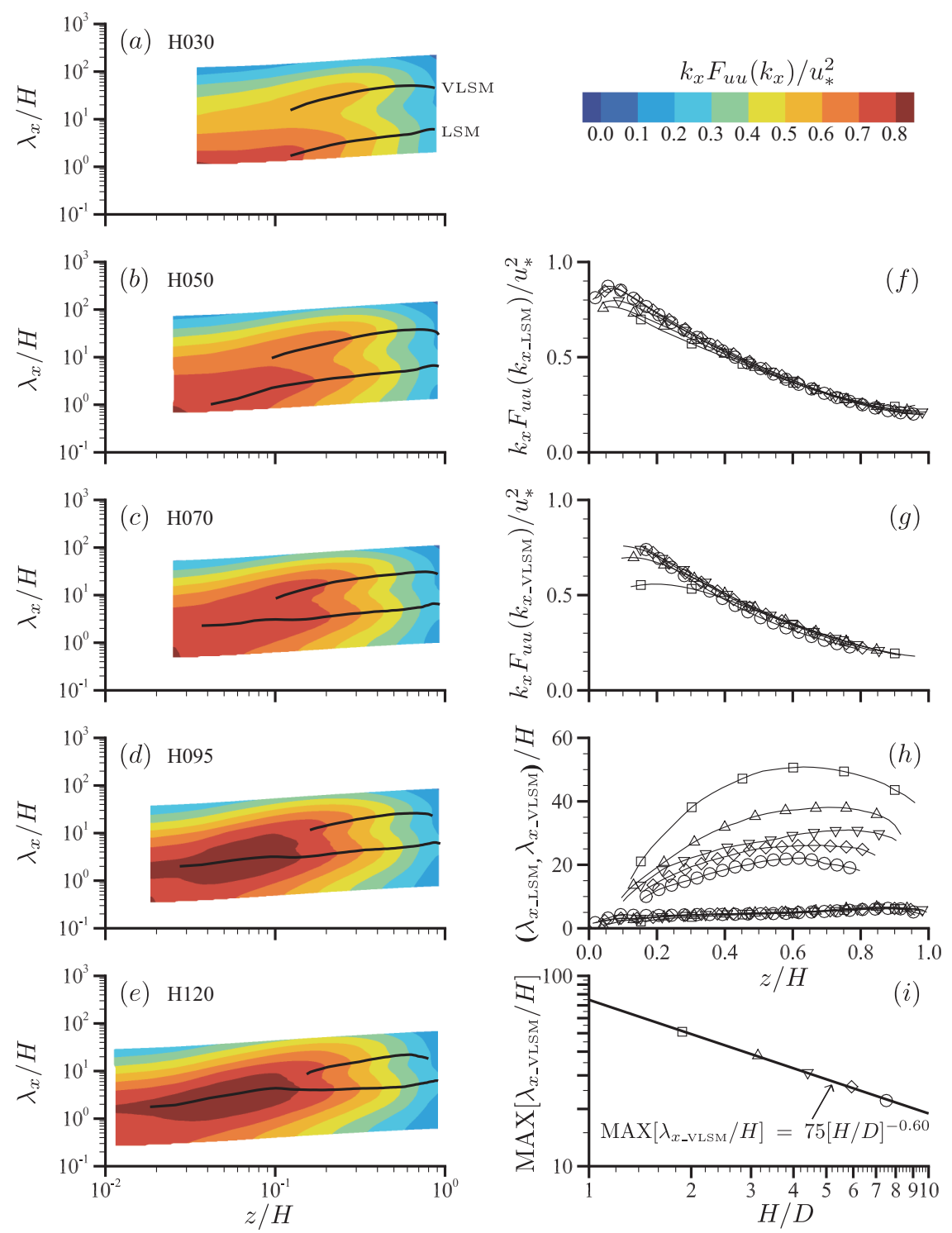

Fig. 5. Pre-multiplied spectra (a-e); amplitude of the LSM (f) and VLSM (g) spectral peaks; wavelengths of the LSM and VLSM peaks (h); maximum wavelength of the VLSM versus flow relative submergence (i) Symbols defined in Figure 2.

\section{Conclusions}

Our measurements show that the pre-multiplied spectra in rough-bed open-channel flows exhibit a bimodal shape in the outer flow corresponding to the presence of large and very large scale motions. The latter motions are also evident as large scale streaks in 'instantaneous' streamwise velocity fields. Two-point correlations suggest that VLSMs may be associated with repeating depth scale counter-rotating cells reminiscent of and 
potentially related to secondary currents in the mean (time-averaged) flow. The streamwise length scale of the LSM collapses across experiments when normalised with the flow depth. However, the depth-normalised VLSM length scale does not collapse. Instead, it exhibits a power-type dependence on either Reynolds number $H^{+}$, relative submergence $H / D$, or flow aspect ratio $B / H$. Since in our experiments we kept channel width, particle size, and shear velocity constant, we cannot unambiguously identify which particular factor $(H / D, B / H$, or $H^{+}$) is responsible for the variation of the depth-normalised VLSM length scale. Supplementary data over self-affine fractal roughness suggest that the development length of VLSMs $(x / H)$ may also play an important role. Differences in the scaling of LSMs and VLSMs suggest that these two motions may form independently of each other.

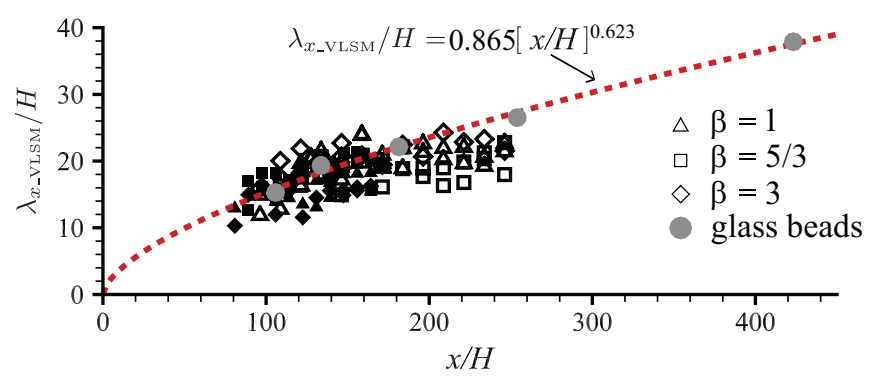

Fig. 6. Development of the VLSM length scale along the channel for self-affine fractal roughness $(\beta=1,5 / 3,3$; filled symbols H120, open symbols H080). Data for glass bead roughness measured at fixed $x$ coordinate but varying $x / H$ is shown for comparison. All points are for $z / H=0.3$. The dashed line is a power law fit to the glass bead data.

The study has been supported by two EPSRC/UK grants, "High-resolution numerical and experimental studies of turbulence-induced sediment erosion and near-bed transport" (EP/G056404/1) and "Bed friction in rough-bed free-surface flows: a theoretical framework, roughness regimes, and quantification" (EP/K041088/1).

\section{References}

1. K. C. Kim, R. J. Adrian, Phys. Fluids, 11, 417-422 (1999)

2. Y. Hwang, C. Cossu, Phys. Rev. Lett. 105, 044505 (2010)

3. N. Hutchins, I. Marusic, J. Fluid Mech. 579, 1-28 (2007)

4. J. P. Monty, N. Hutchins, H. C. H. Ng, I. Marusic, M. S. Chong, J. Fluid Mech, 632, 431-442 (2009)

5. S. M. Cameron, V. I. Nikora, M. T. Stewart, J. Fluid Mech. 814, 416-429 (2017)

6. M. T. Stewart, S. M. Cameron, V. I. Nikora, A. Zampiron, I. Marusic, J. Hydraul. Res. (in print)

7. T. Astarita, G. Cardone, Exp. Fluids 38, 233-243 (2005)

8. I. Nezu, H. Nakagawa, Turbulence in Open Channel Flows (Balkema, 1993)

9. R. J. Adrian, I. Marusic, J. Hydraul. Res 50, 451-464 (2012)

10. B. J. Balakumar, R. J. Adrian, Phil. Trans. R. Soc. A. 365, 665-681 (2007) 